\title{
Responsabilidad en la labor que asumió. Un requisito para las enfermeras en el cuidado humanizado
}

\author{
Oscar Alberto Beltrán Salazar*
}

RESUMEN

Objetivo: Comprender el significado de la experiencia de cuidado de enfermería humanizado para pacientes, familiares y enfermeras. Metodología: Estudio fenomenológico interpretativo que incluyó a 16 participantes adultos y se basó en entrevistas en profundidad para la construcción de la información. Resultados: La responsabilidad de las enfermeras en el cuidado es un requisito para pasar de la atención impersonal al cuidado humanizado y se refleja en el interés por actuar bien y hacer bien las cosas, en lograr las habilidades comunicativas y relacionales, en basar las actuaciones en enfoques teóricos y en estudiar para conocer sobre nuevas orientaciones y prácticas en el cuidado. Conclusiones: La concordancia en las enfermeras entre saber lo que se hace y actuar con orientación al bien favorece el cuidado humanizado.

Palabras clave: Humanización de la atención, cuidado de enfermería, significado, fenomenología.

\section{Responsibility for the assumed work. A requirement for nurses in humanized care}

\begin{abstract}
Objective: To understand the meaning of the experience of humanized nursing care for patients, families and nurses. Methodology: Interpretative phenomenological study involving 16 adult participants, based on in-depth interviews for building information. Results: The responsibility of nurses in care as a requirement to move from an impersonal attention to a humanized care is reflected in the interest in acting well and doing things well, achieving communicative and relational skills, in performing care based on theoretical proposals, in studying and learning about new approaches and practices in the care. Conclusions: The nurses' concordance between knowing what to do and acting with orientation towards the good contributes to humanized care.
\end{abstract}

Key words: Humanization of care, nursing care, meaning, phenomenology.

\section{INTRODUCCIÓN}

$\mathrm{L}$ a forma como se lleve a cabo el cuidado de enfermería es responsable de la forma como es vivido y percibido por los pacientes y sus familiares; por este motivo, las fallas en la labor de las enfermeras que impiden que el cuidado sea humanizado pueden ser responsables en gran parte del malogro de los objetivos terapéuticos. Watson ${ }^{1}$ plantea una categoría denominada "no cuidado", la cual contrasta con el cuidado. Respecto a este último, afirma que está basado en "una relación transpersonal que depende de un compromiso moral para proteger y

* Magister en Enfermería, aspirante a Doctor en Enfermería. Profesor titular de la Facultad de Enfermería. Universidad de Antioquia, Colombia.

Recibido para publicación: 06/05/2014. Aceptado: 19/05/2014.

Correspondencia: Oscar Alberto Beltrán Salazar. Calle 26 Sur Núm. 43 A 41, Apto 731. Villa Santa Teresa, Envigado, Colombia. Tel: 574 2196350, E-mail: oscar4242@udea.edu.co

Este artículo puede ser consultado en versión completa en http://www.medigraphic.com/enfermerianeurologica 
resaltar la dignidad humana, en la cual a las personas se les mire como seres humanos y se les permita determinar sus propios significados", condición que no está presente en el "no cuidado".

Variados estudios muestran las dificultades que se tienen en la realidad para asegurar las condiciones ideales del cuidado de enfermería; por este motivo, Poblete y colaboradores $^{2}$ afirman que "se ha observado en Latinoamérica la necesidad de buscar el significado que tiene el cuidado para las personas y los aspectos que muestran deficiencias en su aplicación, como son el cuidado centrado en el paciente, que éste sea humanizado y que ambos conceptos se incorporen a la praxis"; también plantean que "los estudios revelan que los pacientes valoran más los aspectos del cuidado que tienen relación con la comunicación y el afecto durante su estancia en las instituciones hospitalarias, es decir, cuando el cuidado es humanizado y transpersonal". ${ }^{2}$ Además, afirman los autores que "es difícil mantener los valores humanitarios en el acto de cuidar en las instituciones públicas de salud, en donde parecen invisibilizarse los cuidados de enfermería por las labores biomédicas, pero las enfermeras deben mantener, aún entonces, las virtudes de comunicarse con el otro, ayudarlo y sostenerlo en la difícil etapa de su vida"2 que representa la enfermedad.

Henderson y colaboradores ${ }^{3}$ reportan que a pesar de que las enfermeras están encargadas de velar por la integridad de los pacientes, ellos se sienten desamparados y se quejan cuando sienten amenazada dicha integridad, porque debido a múltiples factores burocráticos y a la sobrecarga de trabajo, el tiempo que las enfermeras interactúan con ellos se reduce notablemente, lo que impide una adecuada interacción enfermera-paciente; entonces, los paciente perciben que las enfermeras no están disponibles para responder a sus solicitudes; en estas condiciones, consideran el cuidado como deshumanizado y que hay cierta devaluación de ellos en su condición de seres humanos. En el mismo sentido de la queja de los pacientes, Forsyth y MacKenzie ${ }^{4}$ reportan que, a su vez, las enfermeras se quejan de la falta de oportunidades para ofrecer un verdadero y comprensivo cuidado a los pacientes, lo que -entre otros motivos de frustración- ha conducido a las enfermeras a retirarse de su labor; por ello, consideran importante que se analicen las condiciones en las cuales trabajan las enfermeras y se realicen los correctivos necesarios para que el desempeño de su trabajo esté de acuerdo con lo esperado.

Con el fin de conocer el significado que tiene para los pacientes, los familiares y las enfermeras el cuidado humanizado, se realizó el presente estudio, guiado por la preocupación que es compatible con la expresada por
Roy y colaboradores, ${ }^{5}$ quienes plantean que a pesar de la "rica historia de desarrollo del conocimiento", sigue habiendo ambigüedad sobre el foco central unificador de la disciplina; por este motivo, es imperativo definir y articular claramente lo que somos y lo que ofrecemos en este momento de nuestra historia. La confusión acerca de un enfoque unificador central es un problema significativo para la práctica, dado el entorno sanitario actual y los problemas mundiales que afectan a la salud y la curación; ${ }^{5}$ también es fundamental ser capaces de "explicar los conceptos básicos y sus interrelaciones que se derivaron de nuestras experiencias y una síntesis de la literatura teórica de la enfermería". Los autores resaltan la importancia del cuidado humanizado al proponer unificar la disciplina en torno a un foco integrador que -según ellos- debe ser "facilitar la humanización, los significados, las decisiones, la calidad de vida y el cuidado en la vida y muerte". 5

El presente estudio no pretendió humanizar el cuidado de enfermería, que de hecho y por definición debe serlo; tampoco realizar una definición de dicho fenómeno, sino que buscó establecer el sentido del cuidado humanizado en la experiencia de las personas que participan en él, mediante la exploración de su significado con una metodología fenomenológica que permitió "mirar la experiencia desde afuera pero involucrarse en ella"; 6 es decir, comprender las experiencias de cuidado humanizado, revisar su significado en la visión de los participantes y reflexionar sobre lo que debe ser y será e, incluso, sobre lo que falta y las desviaciones que han impedido lograr un consenso en la práctica acerca de este fundamental atributo del cuidado. "Comprender" se entiende tal como es descrito por Levinas, ${ }^{7}$ como un acto de existencia según el cual la comprensión de las experiencias ayuda a comprenderse uno mismo.

La mirada fenomenológica condujo a una profunda reflexión sobre aspectos del cuidado presentes en las descripciones que los participantes en el estudio realizaron sobre las experiencias de cuidado percibidas como humanizadas según las condiciones en las cuales se vivieron, el tipo de interacciones con las enfermeras y las relaciones con las instituciones de salud. El fenómeno representado por el cuidado humanizado tiene sentido en la medida en que es conocido por los sujetos, y para lograrlo, se identificaron los comportamientos, las respuestas, las emociones y las motivaciones, de la misma manera como fue posible aclarar e identificar conceptos y presuposiciones que se consideraban poco claras en relación con dicho cuidado.

\section{METODOLOGÍA}

Estudio con enfoque fenomenológico, que incluyó a 16 personas adultas entre 29 y 62 años de edad, cuatro hombres y 
12 mujeres. Seis de ellos eran profesionales que habían tenido experiencia como pacientes en el pasado cuando fueron hospitalizados por situaciones de urgencia, enfermedad crítica o para ser intervenidos quirúrgicamente. De los participantes, siete eran familiares cercanos de pacientes hospitalizados y otros tres eran enfermeras, quienes aceptaron voluntariamente su participación sin recibir estímulos económicos. El muestreo con propósito ${ }^{8}$ permitió seleccionar los mejores informantes para los requerimientos del estudio.

La técnica para lograr la información fue la entrevista conversacional en profundidad, cuya duración fue entre una hora y hora y media. Las entrevistas fueron identificadas con un código para preservar la confidencialidad; en dicho código, se adoptó la letra N seguida de las iniciales del nombre para identificar a las enfermeras participantes, la letra $\mathrm{P}$ para los pacientes y la letra $\mathrm{S}$ para los familiares de pacientes. Los participantes fueron invitados a compartir sus experiencias en relación con el cuidado en sus propias palabras y organizando la descripción en la forma de su elección. La pregunta inicial, "Describa cómo fue su experiencia en el cuidado durante la hospitalización por episodio o episodios de enfermedad propio(s) o de un miembro de la familia", para el caso de los pacientes y familiares y "Describa su experiencia como cuidador con personas enfermas y familiares en el hospital", para las enfermeras, invitó a las personas a realizar su descripción de la experiencia; las preguntas subsiguientes, que fueron específicas para cada una de las entrevistas y dependientes de los contenidos de la información, surgieron de acuerdo con lo expresado para aclarar conceptos manifestados. La pregunta "¿Qué es para usted haber sido cuidado o cuidar humanizadamente en el ambiente hospitalario?" fue la que motivó a hablar sobre los significados del fenómeno en forma específica. Un relato de los hechos durante las interacciones de cuidado, que incluía los pensamientos, sentimientos, emociones, respuestas y comportamientos que tuvieron lugar durante la experiencia de hospitalización, fue importante para comprenderla, así como las percepciones y auto-interpretaciones realizadas por los participantes. También se buscó que se expresaran las percepciones sobre las condiciones del cuidado en relación con las instituciones de salud donde se vivió la experiencia.

El análisis de la información se realizó en forma manual con base en los procedimientos interpretativos de la fenomenología hermenéutica propuestos por Cohen, Kahn y Steeves $;{ }^{9}$ desde la primera entrevista, se inició el análisis al escuchar y reflexionar sobre lo que se expresaba con el fin de captar significados que se pudieron validar con el participante mismo; se transcribieron fielmente las entrevistas y, a continuación, se realizó una inmersión en la información mediante la lectura reiterada, línea por línea, para tener una visión general de lo que se reportaba y de las particularidades de cada experiencia y para lograr un movimiento dialéctico entre el todo y las partes. El producto de esta revisión fue la codificación, el análisis temático y la determinación de unidades de significado y de ejemplares -aquellas porciones de texto con información textual-; esto permitió identificar la presencia de incidentes recurrentes o de temas comunes, así como los casos atípicos o negativos que no encajaban en la línea interpretativa y mostraban variaciones en el análisis; después, los temas y subtemas que fueron significativos en las entrevistas se separaron y se realizaron memos analíticos y diagramas sobre cada uno y sobre las relaciones entre ellos; paso seguido, se realizó una narrativa que describió la forma como se comprendían los temas en relación con la experiencia de los participantes. La interpretación fue validada permitiendo a varios de los participantes leer la descripción para determinar su correspondencia con lo que ellos querían decir, asegurar la fidelidad y credibilidad en el análisis, rescatar lo omitido, favorecer las inferencias y mejorar la descripción final y la validez interna. ${ }^{10}$ Estos textos, producidos a partir de la interpretación de los datos, también fueron revisados por el asesor con título doctoral y amplia experiencia investigativa y 10 miembros del grupo de investigación "Emergencia y desastres" de la Facultad de Enfermería de la Universidad de Antioquia, quienes hicieron sugerencias y recomendaciones que fueron tenidas en cuenta para mejorar el informe y para favorecer la validez externa y la confirmabilidad. A continuación, se realizó la contrastación de los resultados con la literatura y la discusión. Para contribuir a la aplicabilidad del estudio, se presentaron los resultados en diferentes audiencias, lo que también contribuye a la transferibilidad. En todas las entrevistas se consideraron de igual importancia los datos suministrados, sin darles preponderancia por razones de poder social, riqueza, nivel educativo o importancia política de quien los expresaba. El estudio se llevó a cabo en Medellín, Colombia, entre diciembre de 2012 y marzo de 2013. Para su realización se contó con la aprobación del Comité de Ética de la Facultad de Enfermería de la Universidad de Antioquia (Acta CEI-FE 2012-4).

\section{RESULTADOS}

\section{Responsabilidad en la labor que asumió}

Según las descripciones de los participantes, el papel que desempeñan las instituciones en la posibilidad de 
poner en práctica un cuidado humanizado es definitivo para promover los esfuerzos humanizadores y motivar en todo el personal asistencial el interés en la adopción de una forma de proceder que siempre esté orientada hacia el bienestar de los pacientes, el cumplimiento de principios éticos y la preponderancia del ser humano sobre las enfermedades, los procedimientos y los costos. Sin embargo, las personas entrevistadas también hacen referencia a la importancia que le conceden al hecho de que las enfermeras llevan a cabo una práctica real y pertinente de la enfermería basada en sus postulados disciplinares y, en consecuencia, logren aproximarse a un cuidado que pueda llamarse, en toda la extensión de la palabra, de enfermería:

"Humanizar el cuidado, para mí, yo creo que es fácil si no tuviéramos las trabas que nos ponen las instituciones y si nosotros estamos convencidos de que queremos ser enfermeros y amamos la enfermería y su riqueza teórica." NJT

Varios aspectos son importantes en relación con el trabajo que desempeñan las enfermeras en la actualidad; el primero, se refiere a la necesidad de humanizar la atención que prestan con miras a dar un verdadero cuidado, tal como es planteado por la disciplina enfermera y que, por supuesto, debe ser humanizado; el segundo aspecto tiene que ver con la identificación de ciertas fallas que se presentan en el ejercicio de las enfermeras y que no han permitido que la atención que ofrecen pueda considerarse "cuidado de enfermería", porque no corresponde a tal, sino que está compuesto de una serie de acciones descontextualizadas y aisladas donde el paciente no es el protagonista como lo proponen algunas autoras, entre ellas, Henderson. ${ }^{11}$ El tercero, y no menos importante, tiene que ver con ciertas características que deben tener las enfermeras, que son dependientes de los patrones de conocimiento personal y ético y permiten tener el acercamiento necesario para brindar cuidado de enfermería, aceptar a las personas y concederles importancia como seres humanos:

"Desde que usted está montado en este barco es porque le gusta y va a satisfacer unas necesidades de las personas, si usted ya satisfizo las suyas; o sea, porque es que uno no va a satisfacer las necesidades del otro si no tiene satisfechas las de uno mismo." NJT

Las descripciones de los participantes dejan ver la necesidad de integrar todos los patrones de conocimiento en la labor diaria de las enfermeras. La importancia del patrón empírico es evidente para guiar lo que se hace, establecer lo que es recomendable para tomar decisiones acertadas y convenientes en cada una de las situaciones que se presentan; este patrón contribuye a la experticia y seguridad en el desempeño de las funciones, porque si se conoce lo que se hace, es posible hacerlo bien. Así mismo, el patrón de conocimiento personal -que, según algunos autores, es el más importante de los descritos por Carper porque permite integrar los demás patrones y enfocar las actitudes e intencionalidades del cuidado- también es reclamado por las personas que han sido enfrentadas a la atención impersonal de enfermería:

"Uno tiene que ser consciente de que el sistema de salud ha hecho que las enfermeras tengan que delegar muchas funciones y que hoy en día la enfermería no se vea como una vocación sino como un negocio y entonces, eso muchas veces, ni siquiera lo hacen por amor a la profesión sino por amor al dinero y al final es el paciente quien sufre, porque son cuidados sin amor y sin paciencia y la enfermería es de paciencia y amor." $S D$

En relación con el patrón ético, también se puede inferir una especie de reclamación por parte de los participantes en el estudio, porque basar la práctica del cuidado en dicho patrón, permite asegurar la mejor manera de llevar a cabo las interacciones propias del cuidado entre las enfermeras y los pacientes:

"Ummm, ¿la ética? En la universidad imparten muchos seminarios de ética a las enfermeras, pero a la hora del ejercicio pleno de la profesión muchas veces se hace uso de ella y muchas otras se olvida y no se tiene, supuestamente, tanta ética." NG

Estas condiciones propias de las enfermeras pueden fortalecerse con la preparación académica, las reflexiones sobre la práctica y el análisis de contenidos de las propuestas disciplinares y de otras disciplinas, como la ética, la antropología y la sociología, pero es evidente que se requiere empeño, dedicación e inversión de tiempo para acompañar y ayudar:

"Sí, considero que es vocación; primero, que quien está cuidando a un paciente lo hace porque lo quiere hacer y si se sometió a esa situación, la tiene que respetar, porque es algo a lo que uno se compromete de por vida; ya llevo muchos años de ejercicio y le puedo decir que esa es la razón de ser, que nos comprometimos a eso, con 
mayor razón a través de los años y los conocimientos adquiridos." NGG

Así las cosas, la capacitación es vista por los participantes como una manera de actualizar los conocimientos, avanzar hacia otros nuevos y lograr nuevas formas de actuar, acordes con las necesidades que surgen con el progreso, con el avanzar de los años y con los cambios sociales, lo cual, de cierta manera, supera lo esperado por la simple vocación:

"Uno tiene que hacer las cosas bien hechas y para eso uno se preocupa por cualificarse, por estar bien y hacer las cosas bien; sería bueno que las enfermeras se capacitaran más, porque hay gente que salió hace 20 años y sigue haciendo las cosas iguales y no se ha preocupado por mirar qué hay de nuevo; actualizarse da herramientas para hacer todo bien." PGM

La capacitación, también, pone al alcance de las enfermeras de servicio propuestas para promover reflexiones que busquen apropiar nuevos temas en relación con los enfoques del cuidado, tales como los derechos de los pacientes, los aspectos culturales y sociales, y las formas específicas de abordar las situaciones individuales de las personas según el tipo de enfermedad y las respuestas a la misma:

"Pienso que es muy importante que el personal debe recibir charlas que no sean muy esporádicas, sino que sean relativamente frecuentes." PJE

La capacitación informal y los estudios de postgrado son vías recomendables para avanzar en el conocimiento e informarse de los nuevos avances y técnicas que se implementan en el cuidado de la salud, y que enfermería debe conocer para adaptar los cuidados necesarios en cada caso. Los conocimientos y la preparación académica son tan importantes como la actitud para cuidar; es decir, lo que se hace y la forma cómo se hace permiten una vivencia del cuidado que en determinadas condiciones es aceptado y bien evaluado, y en otras es criticado y rechazado cuando las interacciones no dan respuesta a las expectativas de los pacientes y sus familias:

"Le preguntan a uno, le hablan y por ejemplo, en la experiencia que he tenido, cuando el paciente es el que está ahí y ellas, las enfermeras, van y es como muy mecánico el asunto." PJE

La forma de llevar a cabo un cuidado de enfermería humanizado refleja el interés en lo que se hace y en hacerlo bien; además, en buscar la capacitación permanente y desarrollar habilidades comunicativas que favorezcan el acceso a las condiciones de los pacientes, la identificación de sus necesidades y la resolución de sus problemas:

"Tener siempre presente qué es lo que van a hacer, hacerlo bien hecho y preocuparse por ello; proponerse atender bien a los pacientes y porque los resultados no sólo estén enfocados a dar cuenta de indicadores, sino que sean buenos, satisfactorios." PGM

Los participantes consideran importantes las habilidades comunicativas de los miembros del equipo de enfermería, debido a que las formas de hablar y expresarse frente a los pacientes y sus familiares pueden tener impactos positivos, como es lo esperado cuando son formas de comunicación adecuadas que contribuyen a la tranquilidad y bienestar; sin embargo, no hay que perder de vista que también producen efectos negativos de acuerdo con las actitudes, el lenguaje y los modales o formas de comunicación no verbal que los pacientes pueden interpretar como señales de rechazo o falta de interés por parte de las enfermeras, guiados por su estado de salud, las molestias que causa la enfermedad y las condiciones de ansiedad y sufrimiento:

"Todos debemos estar preparados y sensibilizados respecto al tipo de personas que cuidamos, para saberle llegar a la gente, a los que están más sensibles o más susceptibles o a los que están renuentes; yo no digo que para acercamientos dirigidos a entablar amistades, pero sí para conocer y ayudar." NA

El patrón de conocimiento personal es, en este aspecto de la comunicación con los pacientes, un elemento muy importante, debido a que ciertas actitudes y comportamientos de los cuidadores -en este caso, de los miembros del equipo de enfermería- pueden entorpecer la comunicación, limitar las interacciones y perjudicar los resultados del cuidado. En contraste, algunas actitudes son necesarias para favorecer la comunicación y el acercamiento con las personas:

"Yo creo que algunas formas de ser de las personas, su timidez o falta de conocimientos, no las dejan trabajar bien; ellas piensan que es normal para ellas, pero sea como sea, no se comunican bien, mientras que otras que son más extrovertidas sí saben llegar a los pacientes." NA

Puede no ser fácil logar las habilidades para comunicarse, llegar a las personas, seleccionar las palabras 
que permitan el entendimiento, transmitan calma y tranquilidad cuando sea necesario, y logren el aprendizaje y la comprensión que se requieren para asegurar la participación del paciente mismo y de la familia en la recuperación y bienestar. Sin embargo, algunos errores pueden presentarse asociados a los términos que se usan, en las actitudes, el lenguaje postural, no verbal, que no son bien recibidos por las personas y pueden corregirse para lograr el respeto por la dignidad y los derechos de pacientes y los familiares, lo cual es un requisito indispensable para el cuidado humanizado:

"No igualarse con el paciente y usar un lenguaje comprensible; algunos, por su condición, llegan exaltados, agresivos y con un lenguaje rudo, entonces uno no se iguala con ellos y el propósito es lograr que se calmen.” PGM

Así mismo, para los participantes, el conocimiento mutuo entre enfermeras y pacientes, el tiempo o la duración de los contactos y el grado de confianza que se logre en las interacciones permiten al paciente apreciar las cualidades de la enfermera y hasta evaluar su desempeño; y permiten a la enfermera saber quién es en profundidad el paciente, su familia, y detectar todos los elementos e informaciones necesarias para elaborar los planes de cuidado y llevarlos a cabo, también para fijar y personalizar las metas que se esperan en cada caso y la forma de evaluar su cumplimiento:

"Uno tiene que entender al paciente porque puede estar alterado por su enfermedad, puesto que es el paciente el que sufre; si yo no sé de qué está enfermo debo estudiar, tengo que preguntar, pero siempre estar seguro de lo que voy a hacer y a decir, para no ir a perjudicarlo." PJE

Conocer a los pacientes permite establecer cuáles son los problemas o necesidades que requieren atención inmediata y cuáles no, así como los gustos y preferencias que pueden influir en el cuidado; conocerlos permite reconocerlos e interesarse por ellos y por su situación:

"Se debe tener un conocimiento previo, mirar su historia, conversar con la persona para saber con quién se está tratando; conocer lo que le gusta, la profesión que tiene, la actividad que realiza, incluso su historia familiar sirven para acercarse y para llegar prevenidos." NA

Este conocimiento hace posible reconocer la individualidad de las personas, que indispensablemente ha de ser tenida en cuenta durante el cuidado de enfermería humanizado; esto permite sentir que no se trata a cada persona como si fuera igual a todas, como si la categoría "paciente" igualara a las personas, no sólo por el diagnóstico o la enfermedad que padecen, sino también por sus gustos, sus respuestas, sus preferencias y su historia personal; de esta manera, es posible que el paciente perciba que está ocupando un lugar único, irrepetible, que su experiencia personal con la enfermera es exclusiva y su caso y las acciones que recibe también estén revestidas de una exclusividad que permita evitar los errores, realizar acciones pertinentes y confiables y obtener resultados específicos y personales:

"Los pacientes son seres humanos que requieren de todo el cuidado y que nosotros pongamos todo de nuestra parte, considerándolos como los protagonistas de las acciones de cuidado; estar cerca de ellos, tener conciencia de sus necesidades y acompañarlos." NJT

El conocimiento sobre el paciente también permite adecuar las explicaciones y los momentos educativos, establecer el nivel del diálogo y su contenido, y promover aspectos fundamentales como la ubicación en la realidad, la forma de controlar la enfermedad, de entender y familiarizarse con la situación para enfrentarla mejor y lograr el afrontamiento:

"Explicarle cada situación, así uno crea que no está entendiendo, buscar las palabras o los gestos necesarios; hablarle a pesar de que pueda no estar oyendo o que uno sepa que está inconsciente." NA

Se resalta en las interacciones la centralidad del diálogo, que permite captar la proximidad con las personas y promueve la confianza del paciente al sentir que alguien está cerca cuando es necesario; la proximidad, las explicaciones y las actividades educativas relacionadas con la enfermedad, el tratamiento y el rol que los pacientes deben desempeñar para recuperar el bienestar, ineludiblemente deben incluir a los familiares y personas cercanas que requieren explicaciones y entrenamiento respecto a dudas e inquietudes y a la forma como deben integrarse en el cuidado del paciente:

"Desde mi punto de vista personal, considero importantísima a la familia, la enseñanza que necesitan, porque en mi experiencia las familias son muy angustiadas y demasiado preocupadas y también pienso que involucrar a la familia es una forma de acercarse al paciente; hay que explicarles con palabras sencillas y ayudarles a calmarse." NA 
En esta forma de enfocar el cuidado, los indicadores de calidad y humanización pueden ser más flexibles y basarse en las expresiones de agrado y satisfacción de las personas por el cuidado recibido y no sólo por índices de complicaciones, morbilidad o eventos adversos:

"Muy importante que logren que el paciente pueda decir: es que fulanita es muy querida y siempre me trata muy bien; recomiendo mucho preocuparse por la calidad y estar enamoradas de su profesión." PJE

En el otro polo del cuidado humanizado, los participantes en el estudio reportan la existencia de orientaciones al cuidado no humanizadas, que reflejan enfoques individuales de las enfermeras más que lineamientos disciplinares, centradas en los procesos y conocimientos técnicos, que no favorecen el clima de confianza mutua en las relaciones con los pacientes ni benefician el proceso de cuidado y sus resultados; por el contrario, favorecen las interacciones basadas en el temor y en la desconfianza:

"Uno ve que a veces tiene que pagar promesas arrodillado en dos corozos para saber en manos de quién cae, porque puede ser una persona que no sea cualificada y que, obviamente, hace las cosas de mala gana debido a que está insegura." PGM

La confluencia de los enfoques individualizados y los problemas actitudinales da como resultado una práctica del cuidado por parte de las enfermeras que es fraccionada, orientada a tareas y que desconoce la integralidad del ser humano y de los procesos que se orientan a contribuir al bienestar de las personas, que supone un trabajo en todas las esferas, para no reducirlo y parcializarlo:

"Dentro de la función que ellas tienen con los pacientes no está hablar con ellos, sino que es ir, ponerles las inyecciones o ir a cambiarles el pañal o cualquier cosa que haya que hacer." PJE

En este marco de ideas, con una oferta de cuidado exento de condiciones como una verdadera comunicación, se restringe la labor de enfermería a cumplir las órdenes que se relacionan con las actividades del médico y de otros profesionales, y posiblemente se descuidan otras que, por partir de una valoración de enfermería y de la determinación correcta de los problemas, no se realizan en forma responsable. Por lo tanto, no se satisfacen las necesidades o se hace parcialmente y con deficiencias:

"Yo pienso que si estando la familia, de pronto hay negligencia, dígame por ejemplo, en el caso de que no dejen ingresar a la familia y por lo tanto ellos no puedan estar al cuidado del paciente, pienso que es muy posible que lo dejen mojado 4 o 5 horas o lo cambian por allá, no sé, cada que cambien el turno." SDE

Frente a este tipo de práctica no se hacen esperar las reacciones de los pacientes y sus familiares, quienes expresan su insatisfacción con un reclamo para que la práctica del cuidado se revise, se analicen los aciertos y las desviaciones, y se oriente de manera que ofrezca una mejor respuesta a las necesidades de los pacientes y sus acompañantes en condiciones humanizadas y con criterios de calidad más firmes:

"Yo pienso que eso nace más como de una crítica que hacen los mismos pacientes, que dicen que las enfermeras no se acercan, no se toman suficiente tiempo para estar con nosotros y conversar sobre mi diagnóstico, mi pronóstico, mi enfermedad y mi tratamiento.” $N G$

La necesidad de un esfuerzo transformador y humanizador en la práctica de la enfermería es evidente para los participantes en el estudio, así como ajustar las orientaciones laborales al propósito de la enfermería y ofrecer un verdadero servicio a las personas que solicitan el cuidado y a sus familias:

"Entonces es un llamado a toda enfermera para que siempre sea responsable con lo que asumió.” PJE

El cuidado humanizado de enfermería evitará la presencia de la atención impersonal cuando se logre una decisión y un propósito de grupo en el que estén involucrados todos los miembros del equipo de enfermería e incluso otros profesionales, con el apoyo de las instituciones. Se precisa dejar de lado la atención basada en rutinas, en tradiciones y en posiciones personales para adoptar formas de actuar con base en las necesidades reales, ubicadas en el contexto y en un engranaje que permita a todos desempeñar sus funciones coordinadamente, con propósitos comunes, orientados al paciente y su familia:

"El trato al paciente puede ser adecuado tanto como las expectativas del personal de salud lo permitan; depende de si les interesa, porque uno ve que hay ciertas personas que sólo se dedican a realizar acciones aisladas.” NJT

\section{DISCUSIÓN}

Los resultados de este estudio mostraron la importancia de una práctica de cuidado basada en planteamientos 
disciplinares que ofrezcan un marco contextual, base para las reflexiones, para revisar y evaluar teorías anteriores, lo que permitiría que el cuidado se enriqueciera con las múltiples propuestas teóricas existentes y podrían lograrse los mejores resultados; en este aspecto, Duran ${ }^{12}$ plantea, en relación con la enfermería, que "la naturaleza de la práctica disciplinar sugiere que ésta debe ser guiada por teorías científicas. La idea de que la práctica y la teoría están separadas es errónea; más bien hay una estrecha relación entre la práctica y la teoría; porque la segunda le da forma a la primera, a la vez que estructura la investigación”. Para la autora, "la teoría y la práctica no son entidades diferentes, más bien, son diferentes aspectos del mismo fenómeno". ${ }^{12}$ Lo que parece importante, en el estado de desarrollo de la ciencia de enfermería, es que las estructuras paradigmáticas y teóricas presentan una nueva perspectiva para la consideración de los fenómenos y los problemas relacionados con el dominio de la disciplina, y aspectos tan importantes como el bienestar, la salud, la evolución de los procesos de vida y otros se han legitimado y son aceptados como elementos de estudio inherentes a la enfermería. ${ }^{13}$ Éstos, de ser estudiados y retomados en la práctica del cuidado, permiten comprender mejor las experiencias de los pacientes y los familiares, guiar las acciones y una mayor contribución a la solución de los problemas, lo que no puede lograrse con tareas aisladas y descontextualizadas o basadas simplemente en el enfoque biomédico, renunciando a la riqueza y profundidad de la disciplina enfermera.

A propósito de estos planteamientos, Fawcett ${ }^{14}$ propone, a manera de ejemplo, que "el baño en cama ha sido declarado una actividad demasiado simple y poco importante para las enfermeras registradas"; por lo tanto, las auxiliares de enfermería o personal de apoyo ahora realizan lo que antes se pensaba que era una actividad "sagrada" que fomentaba la intimidad entre la enfermera y el paciente, permitía evaluarlo a fondo, hacer planes para otras intervenciones, iniciar la enseñanza y otras acciones de enfermería. ${ }^{14} \mathrm{El}$ resultado ha sido renunciar a estar presente al lado de la cama del paciente, lo que también ocurrió con múltiples intervenciones propias del cuidado directo de enfermería, muy posiblemente para asumir funciones en escritorios u oficinas que nada tienen que ver con la relación enfermera-paciente y, por tanto, no están relacionadas con el cuidado. Según la misma autora, "algunas enfermeras han declarado que los modelos conceptuales de enfermería son "dinosaurios", irrelevantes para la investigación y la práctica contemporánea, recomendando que los libros sobre los modelos conceptuales de enfermería sean relegados a los polvorientos anaqueles y estanterías de la biblioteca. Cada vez más, las enfermeras están recurriendo a los modelos conceptuales de otras disciplinas como guías para su investigación y su práctica". ${ }^{14}$ Esto refleja una situación que amerita reflexión y un nuevo enfoque porque influye negativamente en el cuidado, trayendo como resultado una atención impersonal muy alejada del verdadero cuidado. "En este marco de ideas, la definición de prioridades realizadas por los enfermeros está basada en las expectativas institucionales $\mathrm{y}$, en consecuencia, se deja a los pacientes al cuidado de auxiliares de enfermería, de los familiares o de ellos mismos, en una especie de descuido intrahospitalario" 15 por parte de las enfermeras, lo que desdibuja, a su vez, el que debería ser el verdadero aporte de las instituciones al cuidado humanizado.

Nelson y Gordon ${ }^{16}$ también hacen referencia a la relación entre conocimiento y práctica en enfermería al afirmar que "a pesar de la vasta información de la disciplina, las enfermeras hacen poco uso de ella y prefieren hacer uso de las virtudes y no del conocimiento, incluso acogiendo como propio el conocimiento de la medicina, mientras que el conocimiento de enfermería se tilda de "mere skill" o "tedious drudge work" (simple habilidad o trabajo esclavo tedioso) que no requiere conocimiento especial". ${ }^{16}$

Dos formas de respuesta que se encuentran referenciadas pueden citarse a manera de ejemplo para ilustrar el impacto que producen en las personas las formas de llevar a cabo el cuidado. En primer lugar, cuando los pacientes y familiares no se muestran acordes con la orientación del cuidado, se pueden presentar actitudes de rechazo, que se "relaciona con el tipo de interacciones que establecen las enfermeras, las actitudes que asumen y las respuestas que ofrecen a los pacientes y familiares, quienes valoran los comportamientos y responden con la no aceptación o el rechazo al cuidado, además expresan diversas reacciones y emociones, como rabia y ansiedad" ${ }^{17}$ Comportamientos como "la frialdad, la falta de diálogo y de las explicaciones necesarias no favorecen la aceptación y la confianza hacia la enfermera, pero sí facilitan el rechazo". ${ }^{17}$ En segundo lugar, cuando la forma de cuidar de las enfermeras responde a las expectativas de pacientes y familiares, las manifestaciones "dan la idea de la calidad del servicio", la cual se expresa "con base en el desempeño, las actitudes, las conductas, la experiencia y el conocimiento de las enfermeras, así como por las intervenciones realizadas. También depende del ambiente del servicio, las condiciones ambientales, los tiempos de espera, los elementos tangibles y el tipo de resultados" ${ }^{18}$ En estas interacciones, "la aceptación y la confianza hacia la enfermera no son gratuitas, sino que 
deben ser construidas desde los primeros acercamientos y reforzadas, a su vez, en los contactos diarios". ${ }^{17}$

Lograr la confianza en las relaciones de cuidado trae beneficios tanto para enfermeras como para los pacientes y sus familiares. Hay varios aspectos importantes de considerar en relación con la confianza; el primero es un componente del cuidado descrito por Roach ${ }^{19}$ al afirmar que son necesarios para cuidar "la compasión, la competencia, la confianza, la conciencia y el compromiso". El mismo autor resalta la importancia de la confianza en el cuidado y afirma que "es una cualidad que fomenta relaciones confiadas y favorece la veracidad y el respeto mutuo que son fundamentales para la consecución de los objetivos". La confianza en el cuidado es "recíproca", ${ }^{19}$ se logra y se acrecienta con el contacto directo, sincero y honesto, porque "las dos partes de la relación necesitan sentirse seguras y respetadas". ${ }^{19}$ Sin embargo, también plantea el autor que en la realidad de la práctica de cuidado actual "existen ciertas dificultades porque en muchas instituciones de salud impera la desconfianza por la manipulación de la información", por las deficiencias en el cuidado, en su calidad y humanización; también plantea que en una relación de confianza "no pueden existir ni la coacción, ni la manipulación, ni actitudes paternalistas que anulen la autonomía de las personas". ${ }^{19}$

También se pudo ver en los resultados que para "hacer las cosas bien" es necesario tener preparación y formación, además de buena voluntad e interés, conocer los criterios de calidad para dar respuesta a ella y así mismo, los criterios y requisitos para la humanización si se quiere dar respuesta a esta característica del cuidado. Según Aristóteles, ${ }^{20}$ una persona -y para el caso de este estudio, la enfermera, si se quiere extrapolar el planteamiento del autor- "necesita toda una vida para perfeccionar su quehacer" y "el tiempo es un buen descubridor y coadyuvante para cumplir una función porque conforme avanza, unos y otros van adicionando lo que falta, para lograr el bien de desarrollar la función propia y esto es lo que determina el progreso"; sin embargo, hay que tener en cuenta, en relación con la enfermería, que desde el punto de vista teórico, el avance ha sido inconmensurable, pero desde el punto de vista práctico, aún falta progresar en diferentes áreas y replantear usos y costumbres poco prácticos. Por eso no se ha logrado la excelencia ni la virtud y, por el contrario, las fallas en cuestión de calidad y humanización son notorias, lo que ha obligado a las enfermeras mismas, a las instituciones hospitalarias y a los organismos nacionales e internacionales a proponer medidas para corregir todas las desviaciones que se presentan en la atención de sa- lud y en el cuidado enfermero. Esta misma trayectoria de trabajo arduo y continuo se requiere por parte de las enfermeras para lograr el desarrollo de la interioridad y la capacidad para dar, recibir e interactuar, que tanta falta hacen en la práctica del cuidado

Al considerar y analizar "la parte humana de las enfermeras" en el cuidado, coinciden algunos autores, entre los cuales se puede mencionar a Tschudin, al hablar de la conciencia como atributo del cuidado, respecto a lo cual plantea que "puede definirse como una brújula que dirige nuestra conducta de acuerdo con el estado moral de las cosas; es una instancia fundamental del ser humano que pertenece a su dimensión interior y tiene un valor integrador". ${ }^{21}$ Para Roach,,$^{19}$ por su parte, se puede entender la conciencia como "virtud y no como atributo de la interioridad humana", que hace posible lograr la reflexión en el actuar, la prudencia, la cautela; "el conocimiento de las cosas permite actuar según la recta conducta, es decir, la buena, creativa y compasiva manera de relacionarse".

Respecto a la "parte humana" que se requiere en las enfermeras para el cuidado, juegan un papel importante los patrones de conocimiento definidos por Carper; ${ }^{22} \mathrm{el}$ patrón de conocimiento personal permite "conocerse a sí mismo y actualizar las relaciones auténticas entre la enfermera y el cuidado"; 22 además, hace posible que "el paciente no sea visto como un objeto sino como una persona y permite reconocer que la misma enfermera está siempre aprendiendo y creciendo como persona en una práctica profesional", y se pone en contacto con el paciente mediante interacciones de cuidado. El conocimiento personal es considerado el patrón más importante para "comprender el significado de la salud en términos de bienestar individual". ${ }^{23}$ Las personas vivencian el patrón de conocimiento personal simultáneamente con los otros patrones a medida que participan en su autopreparación ${ }^{23}$ y en las reflexiones sobre el actuar y sobre el conocimiento mismo.

Palazzani ${ }^{24}$ refiriéndose al cuidado y a la importancia de la parte humana de la enfermera al relacionarse con los pacientes -que, en suma, se trata de la humanización de las enfermeras, de su actuar y, por lo tanto, del cuidado-, afirma que este último "se podría definir como actitud interior y compromiso activo de preocupación y atención responsable a quien es vulnerable, en el cual siempre ha existido tensión entre elementos positivos y negativos". ${ }^{24}$

Asumir el gran reto que representa combatir la atención impersonal imperante en las instituciones y favorecida por las enfermeras se apoya en la reflexión sobre muchos aspectos del cuidado, lo que servirá de base para 
lograr el cuidado humanizado que la reemplace; a este respecto, Chesnay ${ }^{25}$ resalta la importancia de enfrentar los cambios sociales, los nuevos riesgos y las variaciones en las poblaciones, al afirmar que "la profesión de enfermería enfrenta retos complejos porque tiene que proveer cuidado a personas y grupos en riesgo. Los roles tradicionales en enfermería y los escenarios de cuidado están cambiando, y de igual forma deben cambiar las respuestas de la enfermería a las necesidades de los diferentes grupos sociales, y estos cambios tienen implicación en los conceptos, las teorías, la ética y la práctica del cuidado". El predominio de la vejez, las enfermedades transmisibles, los cambios ambientales, las altas tempranas y el recorte de los servicios hacen que un nuevo esquema de enfermería sea necesario. ${ }^{26}$ En este marco de acción "la enfermera distante e impersonal, orientada a demostrar habilidad técnica y que no se afecta por el sufrimiento de los demás será reemplazada por una enfermera consciente, que es introspectiva, con capacidad de afrontar y aprender, y con la habilidad para desarrollar relaciones interpersonales, con responsabilidad relacional y honestidad con los pacientes que tienen menos poder que ellas"; dicho de otra manera, "la eficiencia fría será reemplazada con enfermeras que desarrollen intimidad, empatía, relaciones recíprocas con pacientes que son mirados y aceptados como iguales. ${ }^{27}$

\section{CONCLUSIÓN}

Las características personales de la enfermera que conjugan el saber y el hacer o la ciencia y la práctica son esenciales para el cuidado; en este sentido, los enfoques del cuidado obedecen a dos corrientes diferentes: la primera, dirigida a hacer todo bien hecho y hacer el bien, a un ejercicio basado en teorías y planteamientos disciplinares y al desarrollo de habilidades cuidativas y comunicativas; la otra, a poner en acción posiciones personales y enfoques propios que no dan la respuesta esperada por los pacientes, la sociedad ni las mismas enfermeras. En la primera corriente se apoya el cuidado humanizado y en la segunda, la atención impersonal, lo que refleja una forma de lucha interna de la disciplina de enfermería, la cual será resuelta con esfuerzo, interés, capacitación y reflexión constantes.

La responsabilidad en enfermería se refleja en estar pendiente y presente, asumir el rol como enfermeras; además de gustarle lo que hace, tener la vocación y el interés por ayudar con gusto y satisfacción, lo cual es una actitud reconocida por los participantes, como también reconocieron que dialogar, escuchar, comprender, creer la queja e interactuar con afecto y empatía son pilares de la humanización. La formación académica, la experiencia y la actualización contribuyen a la habilidad para interactuar y para asumir una cultura humanizada, que refleje un comportamiento constante y no sólo se oriente a programas, campañas o intenciones temporales y aisladas.

\section{BIBLIOGRAFÍA}

1. Watson J. Nursing: Human Science and Human Care. A theory of Nursing. Boston, Massachusetts: Jones and Bartlett Publisher, 1999. pp. 33-34.

2. Poblete M, Troncoso L, Valenzuela S. Cuidado humanizado: un desafío para las enfermeras en los servicios hospitalarios. Acta Paul Enferm. 2007; 20 (4): 499-503.

3. Henderson A, Van Eps MA, Pearson K, James K, Henderson P, Osborne Y. "Caring for" behaviors that indicate to patients that nurses "care about”. Jour Adv Nurs. 2007; 60 (2): 146-153.

4. Forsyth S, MacKenzie H. A comparative analysis of contemporary nurses 'discontents'. Jour Adv Nurs. 2006; 56 (2): 209-216.

5. Willis DG, Grace PJ, Roy C. A central unifying focus for the discipline facilitating humanization, meaning, choice, quality of life, and healing in living and dying. Adv Nurs Sci. 2008; 31 (1): E28-E40.

6. Heidegger M. Ser y tiempo. México: Fondo de Cultura Económica; 1951.

7. Levinas E. ¿Es fundamental la ontología? Entre nosotros. Ensayos para pensar en otro. Valencia: Pre-Textos, 2001. pp. 13-23.

8. De la Cuesta C. Cuidado artesanal. La invención ante la adversidad. Medellín: Facultad de Enfermería de la Universidad de Antioquia, 2004. p. 38.

9. Cohen M, Kahn D, Steeves R. Hermeneutic phenomenological research. A practical guide for nurse research. Londres: Saac publications, 2002. p. 72.

10. Miles M, Huberman M. Qualitative data analysis. 2nd ed. Thousand Oaks: Sage; 1994.

11. Henderson V. Podemos dar un papel estelar a los pacientes. Inves Educ Enfer. 2000; 28(1): 125-128.

12. Duran MM. La ciencia, la ética y el arte de enfermería a partir del conocimiento personal. Aquichan. 2005; 5 (1): 86-95.

13. Chinn PL, Kramer M. Theory and Nursing: Integrated knowledge development. 5th ed. Saint Louis: Mosby, 1999. pp. 31, 34.

14. Fawcett J. Guest Editorial: On bed baths and conceptual models of nursing. Jour Adv Nurs. 2003; 44: 229-230. Disponible en: doi: 10.1046/j.1365-2648.2003.02823.x. [Access November 2013].

15. Beltrán SO. Dilemas éticos en el cuidado enfermero. Index Enferm. 2011; 20 (1-2): 36-40.

16. Gordon S, Nelson S. Moving beyond the virtue script in nursing. Creating a knowledge-based identity for nurses. In: Nelson S, Gordon S. The complexities of care. Nursing reconsidered. London: IRL press and Cornell University Press; 2006. pp. 13-29.

17. Beltrán O. Rechazo de los pacientes del cuidado enfermero. Invest Educ Enferm. 2011; 29 (3): 343-352.

18. Losada OM, Rodríguez OA. Calidad del servicio de salud. Cuad. Adm. Bogotá, 2007; 20 (34): 237-258.

19. Roach $\mathrm{S}$. The human act of caring: a blueprint for the health professions. Ottawa: Canadian Hospital Association; 1987.

20. Aristóteles. Ética Nicomáquea. Traducción y notas de Palli Julio. Buenos Aires: Planeta DeAgostini; 1995. p. 31

21. Tschudin V. Ethics in nursing. The caring relationship. 2nd ed. Oxford: Butterworth-Heiemann; 1992.

22. Carper B. Fundamental patterns of knowing in nursing. Adv Nurs Sci. 1978; 1 (1): 13-23.

23. Parker M. Nursing theories and nursing practice. 2 nd ed. Philadelphia: FA Davis Company; 2005. 
24. Palazzani L. La contribución de la bioética en femenino a la praxis del cuidado. Azafea Rev Filos. 2008; 10: 145-157.

25. Chesnay M, Anderson B. Caring for the vulnerable. Perspectives in nursing theory, practice and research. 2nd ed. Sudbury: M. Jones and Bartlett Publishers; 2008.
26. Rosa R, Zamora MG. Cuidados invisibles. Son suficientemente reconocidos. Index (gran). 2012; 21 (4): 219-223.

27. Aranda S, Brown R. Nurses must to be clever to care en complexities of care. In: Nelson S, Gordon S. The complexities of care. Nursing reconsidered. London: IRL press and Cornell University Press; 2006. pp. 122-142. 